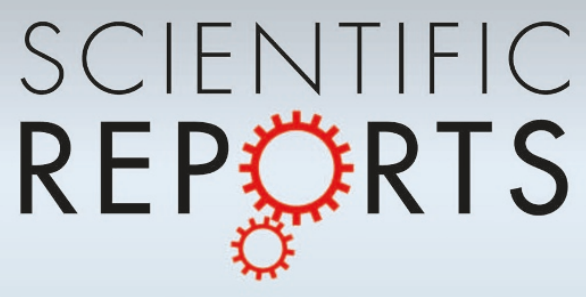

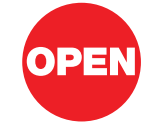

SUBJECT AREAS:

NEURAL STEM CELLS

BIOMATERIALS - CELLS

TISSUE ENGINEERING AND

REGENERATIVE

MEDICINE

STEM-CELL RESEARCH

Received

14 December 2012

Accepted

15 March 2013

Published

3 April 2013

Correspondence and requests for materials should be addressed to

J.W.D. (jwdai@

genetics.ac.cn); M.L.T.

(mltang2010@sinano.

ac.cn) or G.S.C.

lgscheng2006@

sinano.ac.cn)

* These authors contributed equally to this work.

\section{Three-dimensional graphene foam as a biocompatible and conductive scaffold for neural stem cells}

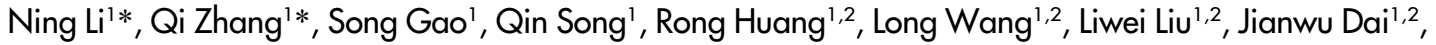 \\ Mingliang Tang' \& Guosheng Cheng ${ }^{1,2}$
}

'Suzhou Key Laboratory of Nanobiomedicine \& Division of Nanobiomedicine, Suzhou Institute of Nano-Tech and Nano-Bionics, Chinese Academy of Sciences, Suzhou 215123, P. R. China, 'niversity of Chinese Academy of Sciences, 19A Yuquan Road, Beijing, 100049, P. R. China.

Neural stem cell (NSC) based therapy provides a promising approach for neural regeneration. For the success of NSC clinical application, a scaffold is required to provide three-dimensional (3D) cell growth microenvironments and appropriate synergistic cell guidance cues. Here, we report the first utilization of graphene foam, a 3D porous structure, as a novel scaffold for NSCs in vitro. It was found that three-dimensional graphene foams (3D-GFs) can not only support NSC growth, but also keep cell at an active proliferation state with upregulation of Ki67 expression than that of two-dimensional graphene films. Meanwhile, phenotypic analysis indicated that 3D-GFs can enhance the NSC differentiation towards astrocytes and especially neurons. Furthermore, a good electrical coupling of 3D-GFs with differentiated NSCs for efficient electrical stimulation was observed. Our findings implicate 3D-GFs could offer a powerful platform for NSC research, neural tissue engineering and neural prostheses.

r he neural stem cells (NSCs) are a self-renewing and multipotent cell population in the central nervous system, which exhibit promising prospects in developing cell therapies for neural regeneration ${ }^{1,2}$. Constructing a microenvironment, a scaffold that regulates NSC behavior and tissue progression has been an essence in clinical applications ${ }^{3}$.

Nowadays, the emerging carbon nanomaterials offer numerous opportunities to design novel scaffolds for neural tissue engineering ${ }^{4}$. Graphene foam (GF), a three-dimensional (3D) porous structure ${ }^{5}$, has been utilized in electronics and energy storage/conversion systems $\mathrm{s}^{6-8}$. In the field of tissue engineering, 3D-GF could be one promising candidate for novel NSC scaffold as it may incorporate topographical, chemical and electrical cues in the same scaffold to provide an environment for neural tissue regeneration that is superior to conventional inert biomaterials:

(1) Directing differentiation. It was reported that the unique surface properties of two-dimensional (2D) graphene can induce stem cell to preferentially differentiate into specific lineage ${ }^{9-11}$.

(2) 3D porous structure. Different from 2D stem cell culture system, the porous GF could provide 3D microenvironments in which cells are able to resemble their in vivo counterparts.

(3) Electrical conductivity. The GFs possess an enormous interface and 3D multiplexing and highly conductive pathways for charge transport ${ }^{5}$. Given neural cells are electro-active and electrical stimulation can affect NSC behaviors ${ }^{12-14}$, it's promising to develop a robust GF-based 3D bioelectronic interface to regulate NSC behaviors.

To date, few studies involved the interaction between flat 2D graphene films and stem cells, while there are scarcely any data on the interaction of 3D-GFs and NSCs as well as the electrical coupling efficiency between the $3 \mathrm{D}$ conductive scaffold and cells ${ }^{9-11}$. In this paper, we demonstrated the first example of $3 \mathrm{D}-\mathrm{GF}$ as a novel scaffold for adult NSCs derived from hippocampus of postnatal day 1 ICR mouse in vitro, and conducted proof-ofconcept studies on the 3D-GFs as a conductive platform for cell electrical stimulation.

\section{Results}

Synthesis and characterization of 3D-GFs. 3D-GFs were synthesized by chemical vapor deposition method using Ni foam template ${ }^{5}$. Scanning electron microscopy (SEM) observation (Fig. 1a and 1b) shows that 3D-GFs 

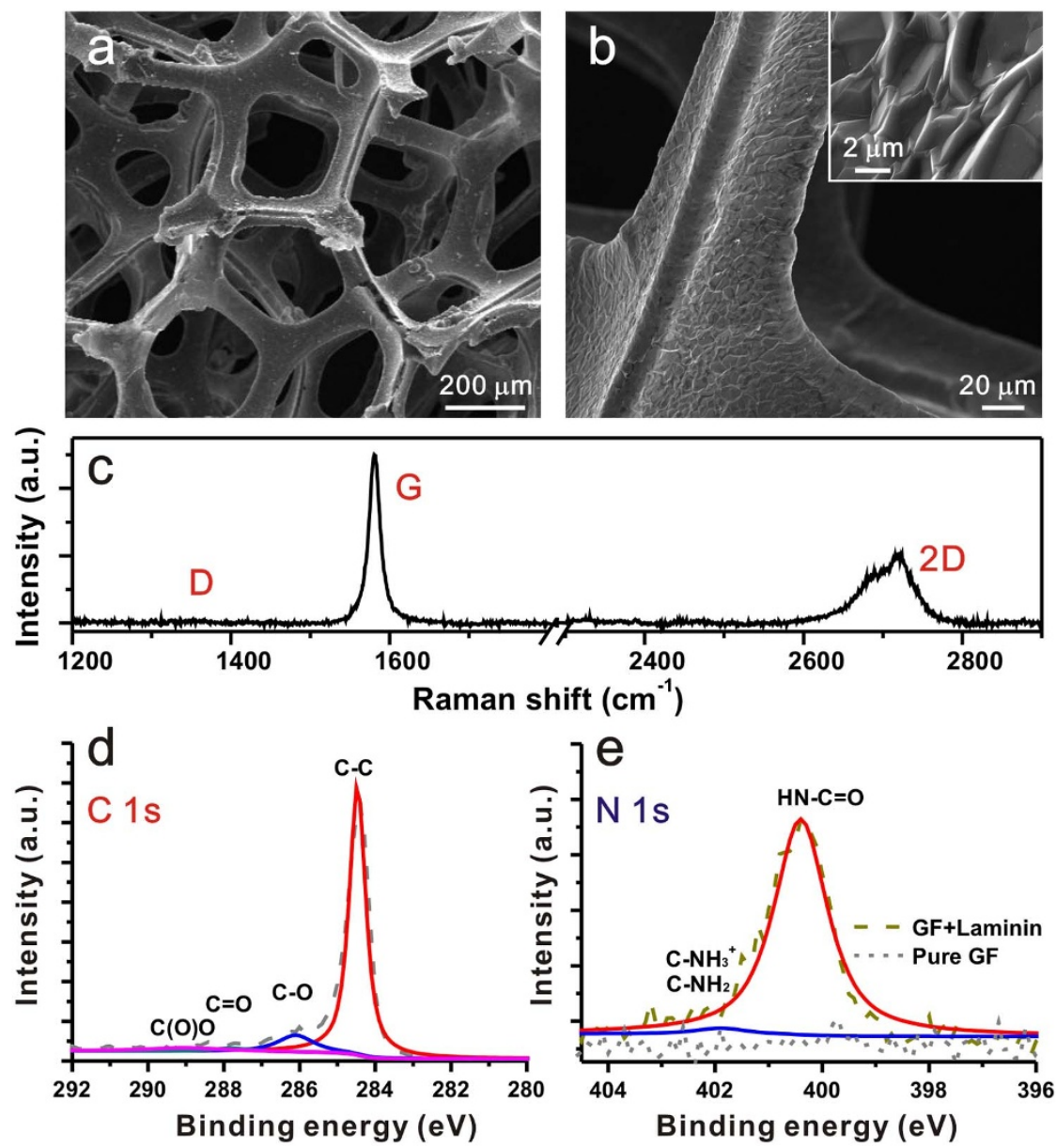

Figure 1 Characterization of 3D-GF scaffold. SEM micrographs of 3D-GFs at low (a) and high (b) magnification, the inset shows an enlarged view of 3D-GF skeleton surface. (c) Typical Raman spectrum of 3D-GFs. (d) High-resolution C (1s) XPS spectra of 3D-GFs. (e) High-resolution N (1s) XPS of 3D-GFs with pre- and post- laminin treatment.

exhibited a monolith of continuous and porous structure, which copied and inherited the interconnected 3D structure of the $\mathrm{Ni}$ foam template. The porosity of 3D-GFs was determined to be 99.5 $\pm 0.2 \%$ and had a pore size of $100-300 \mu \mathrm{m}$, while the width of the graphene skeleton was about $100-200 \mu \mathrm{m}$. 3D-GF surface was covered with many ripples and wrinkles at micro- and nano-scale (Supplementary Fig. S1). Furthermore, the absence of D band in Raman spectrum (Fig. 1c) indicated that 3D-GFs were of high quality with few defects, while the shape of $2 \mathrm{D}$ band and the intensity ratio between $2 \mathrm{D}$ and $\mathrm{G}$ band proved that $3 \mathrm{D}-\mathrm{GF}$ s were constructed by few-layer or multi-layer graphene sheets ${ }^{15}$. Although the graphene layers were extremely thin, the network possesed excellent mechanical strength and flexibility, and can stand alone. Addtionally, compared with the structure of 3D-GFs prepared by freeze-drying or template assembly method, the monolith of 3D-GF network by chemical vapor deposition ensured a high conductivity owing to the lack of defects and inter-sheet junction contact resistance ${ }^{16}$.

The surface chemistry of 3D-GFs was further characterized by the X-ray photoelectron spectroscopy (XPS). In Fig. 1d, two obvious components were observed in the C1s XPS spectrum of pure 3DGFs. The main peak at $284.6 \mathrm{eV}$ corresponds to the non-oxygenated ring $\mathrm{C}$, while a small peak reflects $\mathrm{C}$ in $\mathrm{C}-\mathrm{O}$ bonds, indicating a relative inert surface chemistry. The removal of trace amounts of the catalyst and etching agents was also verified by XPS and revalidated by energy dispersive X-ray spectrum (Supplementary Fig. S2). In addition, lots of protocols for facile chemical modification using natural and synthetic bioactive molecules have been well developed for graphene, which facilitates NSC scaffold functionalization $^{11,17}$. In this work, laminin coating was adapted to enhance cell adhesion. As shown in Fig. 1e, after immersing 3D-GFs in a laminin solution for more than $4 \mathrm{~h}$, there were obviously $\mathrm{N}$ 1s peaks arising in the high-resolution N (1s) XPS spectrum of 3D-GFs, indicating the success of laminin modification ( $\mathrm{N}$ contained in the laminin molecule). Also, morphology of laminin-coated 3D-GFs was almost identical with that of pure 3D-GFs, and no obvious difference between them was observed (Supplementary Fig. S3).

NSC adhesion on 3D-GFs. NSC adhesion on 3D-GFs was firstly investigated. For no more than 10 hours after cell seeding, almost no free-floating cell could be found in the culture medium (data not shown), indicating a rapid cell attachment on the 3D-GFs. After another 5 days of culture, the interaction between NSCs and 3DGFs was examined by SEM. NSCs cultured on 3D-GFs formed a well neural network and exhibited excellent cell adhesion (Fig. 2a \& 2b). High resolution SEM image shows that the cells spread extensively and formed strong filopodia/GF interaction (insert of Fig. 2b). Furthermore, in cross section fluorescence image of 3D-GF scaffold with NSCs (DAPI staining), a number of cells were observed inside the scaffold as well as on the surface, clearly indicating that the cells grew in a 3D fashion (Supplementary Fig. S4). Additionally, the SEM observation presents the $3 \mathrm{D}$-GFs remained intact during cell culture process over 2 weeks.

Biocompatibility of 3D-GFs. 3D-GF cytotoxicity was evaluated by Calcein-AM and EthD-I staining assay with 2D graphene film as 
control. Fig. 2c shows that almost $90 \%$ of the cells cultured on 3DGFs for 5 days were viable, while the difference in cell viability between 3D-GFs and 2D graphene films is neglectable (lower inset in Fig. 2c \& Supplementary Fig. S5). Nor could a TUNEL assay find any abnormal cell apoptosis on 3D-GFs (Supplementary Fig. S6). Those data demonstrated good biocompatibility of 3D-GFs, consistent with previous studies ${ }^{10,11,18}$. The cells were also stained with antibody against nestin, a protein marker of NSCs. Fig. 2d shows that nearly all of cells on 3D-GFs were immunopositive for nestin (green), with no obvious difference from that on 2D graphene films (Supplementary Fig. S5), indicating that NSCs proliferated well on $3 \mathrm{D}-\mathrm{GFs}$ while maintained their stemness.

NSC proliferation on 3D-GFs. NSC proliferation on 3D-GFs was examined by measuring the expression of Ki-67 protein, a cellular marker for proliferation ${ }^{19}$. High specific surface area of 3D-GFs $\left(200-800 \mathrm{~m}^{2} / \mathrm{g}\right)$ could provide a large surface area for cell attachment and growth ${ }^{5}$. Also, macroporous structure of $3 \mathrm{D}-\mathrm{GFs}$ is believed to ensure efficient mass transport of nutrition for NSC metabolic demands, which should facilitate cell proliferation. As
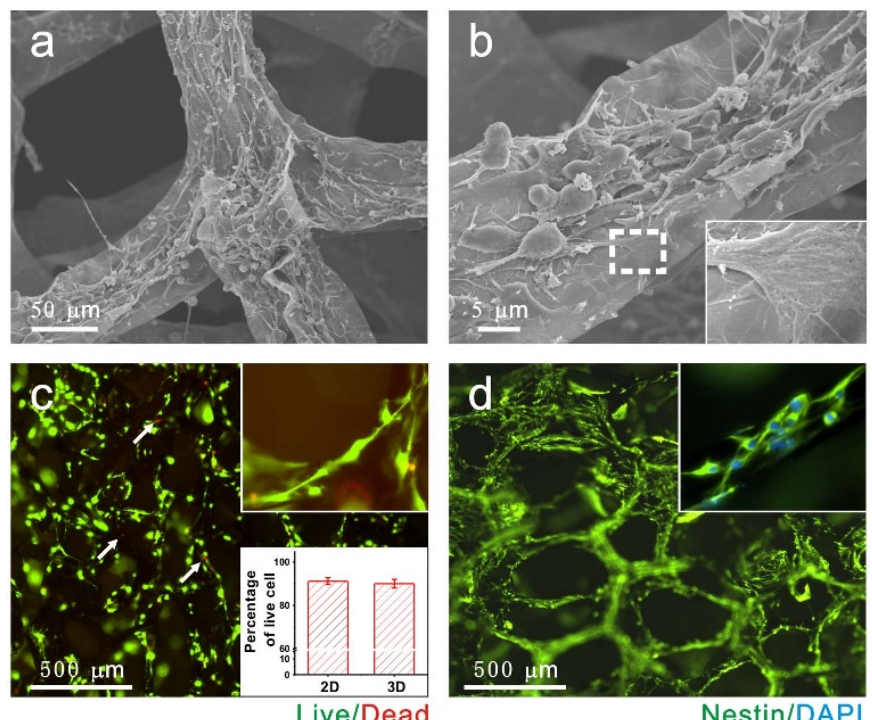

Live/Dead
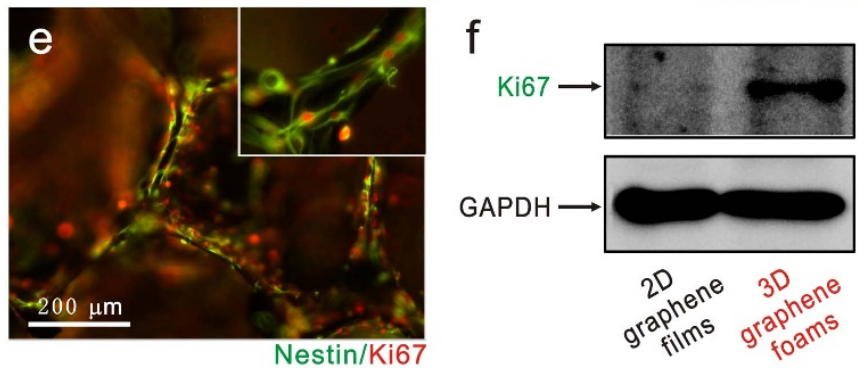

Figure $2 \mid$ NSC adhesion and proliferation on 3D-GF scaffold. Low- (a) and high- (b) magnified SEM images of NSCs cultured on 3D-GFs under the proliferation medium. The inset illustrates the interaction between the cell filopodia and 3D-GF surface. (c) Cell viability assay of NSCs on 3DGFs after 5 days of culture as determined by live/dead assay, live cells are stained green and dead cells are red, white arrow points to dead cell. The lower right inset depicts the percentage of live cell on 2D graphene films and 3D-GFs. (d) Fluorescence images of NSCs proliferated on 3D-GFs for 5 days, immunostaining markers were nestin (green) for neural stem cells and DAPI (blue) for nuclei. (e) NSCs were double-immunostained with anti-Ki67 (red) and anti-nestin (green) antibodies, Ki67 is a marker for cell proliferation. (f) Western Blot analysis of Ki67 expression on 2D graphene films and $3 \mathrm{D}-\mathrm{GFs}$. The Data are presented as mean \pm standard error (s. e. m.), ${ }^{*} p<0.05, * * p<0.01$. expected, Fig. 2e shows that a majority of (nearly 80\%) cells on 3DGFs were stained positively for Ki-67. Further quantitative western blot analysis illustrates that the expression of Ki-67 was significantly higher in 3D-GF groups than in 2D graphene film groups (Fig. 2f), suggesting that NSCs on $3 \mathrm{D}-\mathrm{GF}$ sustained a more active proliferation state (consistent with WST-based cell proliferation assay, Supplementary Fig. S7), which could be of a huge advantage as a scaffold material to increase NSC number after the transplantation ${ }^{3}$.

NSC differentiation on 3D-GFs. The phenotypic changes of differentiated NSCs on 3D-GFs were further investigated. After 5 days differentiation, the cells exhibited elongated cell shape with healthy neurite outgrowth, leading to a confluent neural network covering almost the whole 3D-GF surface (Supplementary Fig. S8). Immunofluorescence staining shows that all Tuj- $1^{+}$(neuron marker), $\mathrm{O}^{+}$ (oligodendrocyte marker) and $\mathrm{GFAP}^{+}$(astrocyte marker) cells were observed on 3D-GFs (Fig. 3a \& 3b) and 2D graphene films (Supplementary Fig. S9), indicating that NSCs keep the pluripotency to differentiate into all three neural subtypes. For a quantitative analysis, cells were harvested and subjected to western blot assay. As shown in Fig. 3c \& 3d, compared with 2D graphene film groups, NSCs cultured on 3D-GFs exhibited tremendously lower nestin expression, while the expression of Tuj-1 and GFAP were enhanced in cells by $\sim 2.5$ and $\sim 1.5$ folds, respectively. Additionally, there was no significant difference in RIP (also oligodendrocyte marker)
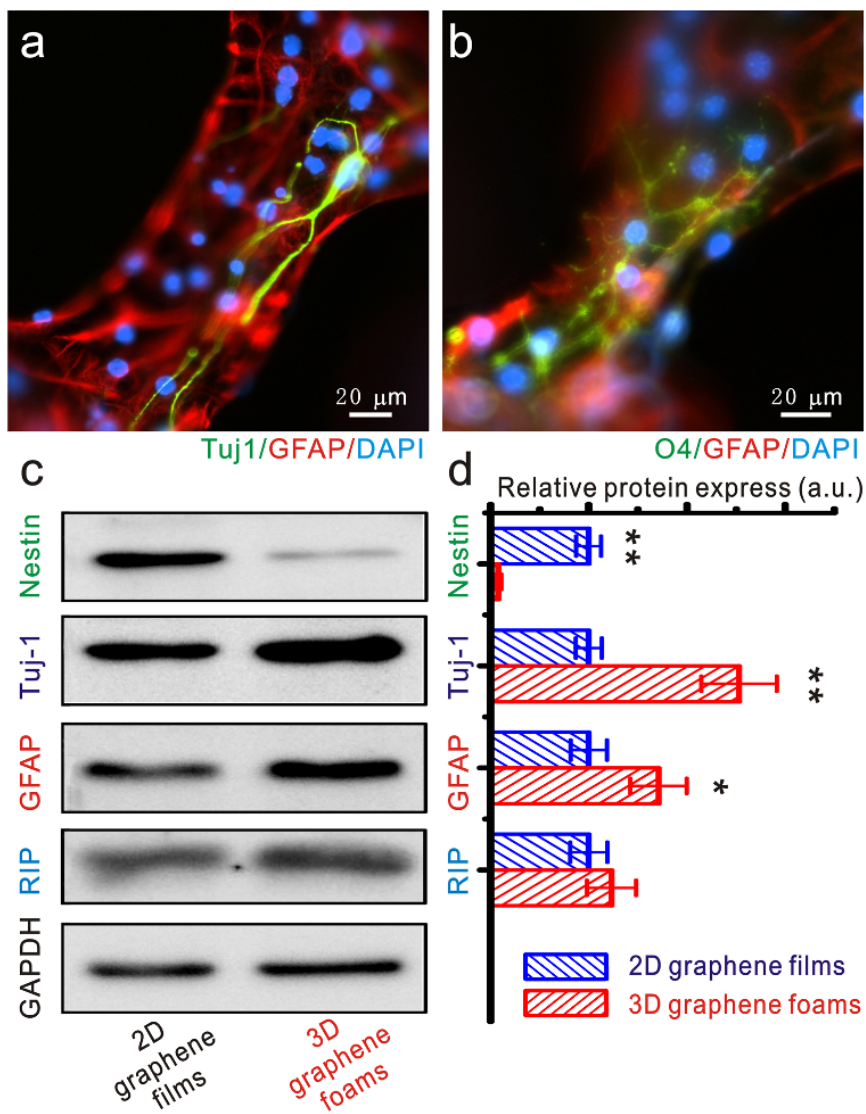

Figure 3 The differentiation of NSCs on 3D-GF scaffold.

(a, b) Representative fluorescence images of differentiated NSCs under differentiation conditions, the cells were immunostained with Tuj-1 for neuron (green, a), GFAP for astrocyte (red, a\&b), O4 for oligodendrocyte (green, b) and DAPI for nuclei (blue, a\&b). (c) Western blot analysis of nestin, Tuj-1, GFAP and RIP protein expression of differentiated NSCs on 2D graphene films and 3D-GFs. (d) Relative optical densities of nestin, Tuj1, GFAP and RIP bands shown in (c). The Data are presented as mean \pm standard error (s. e. m.), ${ }^{*} p<0.05,{ }^{* *} p<0.01$. 
expression between the two experimental groups. The results indicated that 3D-GFs can greatly enhance NSC differentiation into neurons and astrocytes, especially neuronal lineage.

Electrical stimulation. To utilize $3 \mathrm{D}-\mathrm{GFs}$ as a cell stimulation electrode, the electrochemical property of 3D-GF was measured by cyclic voltammetry using a potentiostat with three-electrode system in phosphate buffered solution (PBS). As shown in Supplementary Fig. S10, basically featureless voltammogram curves of the 3D-GF and $2 \mathrm{D}$ graphene film electrode are observed within the operational potential window from $-0.3 \mathrm{~V}$ to $1.0 \mathrm{~V}$, indicating that the current is delivered primarily through charging and discharging the interfacial double layer instead of through faradic reactions. It's indicted that the capacitive charge injection is ideal for neural stimulation since no chemical change occurs to either the tissue or the electrode ${ }^{14}$. Meanwhile, under the same potential, the current of $3 \mathrm{D}-\mathrm{GF}$ electrode is significantly higher than that of $2 \mathrm{D}$ graphene film owing to its higher double-layer capacitance originated from the larger specific surface area, which can lead to a stronger charge injection ability.

Right before electrical stimulation, differentiated NSCs on 3D-GFs were stained with Fluo-4 AM dye to monitor the change of intracellular calcium ion concentrations caused by electrical stimuli. Fluo- 4 $\mathrm{AM}$ is a membrane-permeable, $\mathrm{Ca}^{2+}$ dependent dye and exhibits a large fluorescence intensity increase on binding of free $\mathrm{Ca}^{2+}$. Previous report showed that the voltage pulse stimuli on a neuron could open calcium ion channels and increased the calcium ion concentration of cell, resulting in the enhanced fluorescence intensity of Fluo-4 AM dye in the neuron ${ }^{20}$. In our study, a series of monophasic cathodic pulses were applied using a function generator and the stimulation threshold was $20-30 \mu \mathrm{A}$. Fig. 4 a shows the fluorescence level of the differentiated NSCs on 3D-GFs increased during a stimulus. The relative change in fluorescence intensity $\triangle F / F$ was plotted versus stimulation time in Fig. 4b, the cells exhibited over 50$60 \%$ fluorescence intensity increase by electrical stimuli. The results clearly imply that 3D-GFs can work as a conductive scaffold to electrically stimulate cells.

\section{Discussion}

Cell adhesion, biocompatiblity and other biological effects of a scaffold are essential for its tissue engineering applications. NSCs can
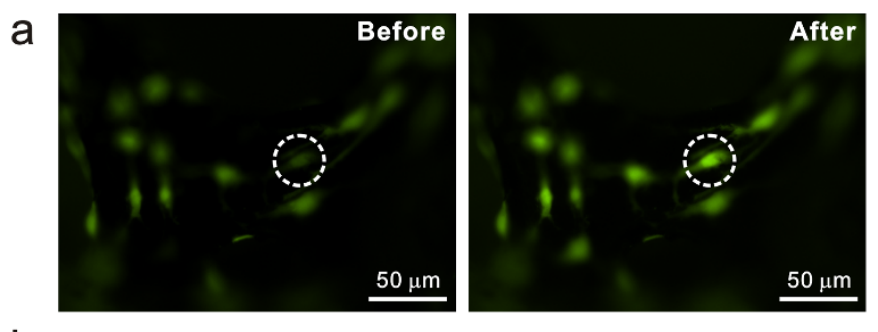

b

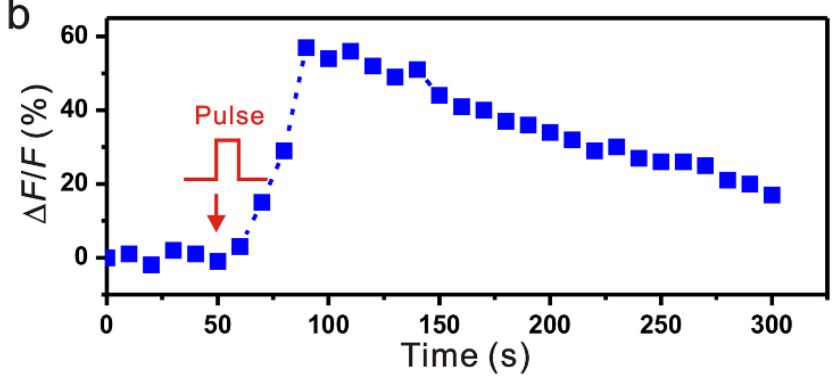

Figure 4 Electrical stimulation of the cells differentiated from NSCs on 3D-GFs. (a) Fluorescence imaging of the cells pre-incubated with Fluo-4 AM dye on 3D-GFs before (left) and after (right) electrical stimulation. Panel (b) plots the relative fluorescence intensity change $\Delta F / F$ of the circled cell in panel (a) versus the stimulation time period. proliferate and differentiate into different cell types when they attach onto a substrate under appropriate conditions, and thus can be considered as anchorage-dependent cells. Anchorage-dependent cells require good adhesion to a substrate in order to spread, proliferate, and maintain cellular functions. In our study, good NSC adhesion can be found for $3 \mathrm{D}-\mathrm{GFs}$ (Fig. $2 \mathrm{a} \& 2 \mathrm{~b}$ ), which is a crucial prerequisite for biological application of 3D-GFs. The good performence of cell adhesion on 3D-GFs, consistent with 2D graphene films for NSCs and human mesenchymal stem cells, should mainly ascribed to unique surface property of graphene materials itself ${ }^{9-11}$. Also, the ripples and wrinkles on the surface of 3D-GFs probably resulted in an improved mechanical interlocking with cells and consequently better adhesion, for surface structure of biomaterial can significantly affect the cell adhesion ${ }^{21}$.

The biocompatibility of graphene could be very tricky owing to the differences of graphene synthesis techniques and the variety of the biological subjects studied. In our experiement, no obvious cytotoxicity of 3D-GFs could be observed for NSCs, as 3D-GFs didn't tremendously affect NSC viability (Fig. 2 and Supplementary Fig. S6 \& S7). The good biocompatibility of 3D-GFs is consistent with the previous studies that $2 \mathrm{D}$ graphene films exhibited as good neuralinterfacing material ${ }^{10,11,18}$. There may be two issues for the friendly interaction of 3D-GFs with NSCs. First of all, chemical vapor deposition method used in synthesis of 3D-GFs could afford high-quality graphene in a relatively pure form ${ }^{10,22}$, which allows the biology effect to be less affected by impurity. Additionally, the coating with laminin (a widely used biomolecule for NSC culture) is a standard protocol for the surface treatment of NSC-related biomaterial, which may also produce a NSC-favorable surface of 3D-GFs. Very recently peptidecoating was reported to offer a more cytocompatible graphene surface for adhesion, spreading of retinal ganglion cells ${ }^{23}$.

Apart from excellent biocompatibility, it is remarkable that $3 \mathrm{D}$ GFs can enhance the NSC differentiation towards astrocytes and especially neurons. The observed effect is almost certainly due to a complex interplay of chemical and physical properties of graphene and cells. The contribution of composition was meticulously considered and firstly excluded. It was well accepted that the impurity of biomaterials can induce biological effects and great efforts were spent on this issue in our experiment. A strict protocol was developed to produce impurity-removed graphene materials. After purification, no detectable amount of catalyst was measured in 2D graphene films ${ }^{18}$ and 3D-GFs (Supplementary Fig. S2 ). Additionally, in the previous reports, there is no evidence that the biological effects of the purified graphene are related to the catalyst metal residue after standard chemical etching, compared with glass, silicon, poly(dimethylsiloxane) (PDMS) and poly(ethylene terephthalate) (PET) materials $^{9-11}$. Hence the difference between $3 \mathrm{D}-\mathrm{GFs}$ and $2 \mathrm{D}$ graphene films should be ascribed to the non-composition factors of 3DGFs. Besides chemical composition, the wettability, stiffness, dimensions, topographical and electrical properties of substrates can affect biological effect of biomaterial. This makes it difficult to identify the microscopic origin of biology effects of 3D-GFs. Fortunately, the comparison experiment does offer some clues: since both 3D-GFs and $2 \mathrm{D}$ graphene films were fabricated from the same material and had similar surface chemistry (Fig. 1 \& Supplementary Fig. S11), the notable difference of NSC differentiation on 3D-GFs and 2D graphene films is more likely due to the different surface morphology and spatial structure. The surface of 3D-GFs possessed micro-scale topographic features (curvature, roughness, anisotropic microstructure) different from graphene films, which would influence NSC behaviors through contact guidance. For instance, the $\mathrm{V}$-shaped channels microstructure on 3D-GF skeletons (Fig. 1) may confine NSC growth and facilitate neuronal differentiation ${ }^{24}$. Meanwhile, GFs exhibit more complex 3D structures with interconnected pores, while graphene films are 2D relatively smooth surface. Cellular communication, transportation of oxygen and nutrients, removal of 
wastes and cellular metabolism on 3D-GFs are more efficient than those on 2D graphene films.

One new trend of scaffold design is to create conductive platform that introduces external electrical stimuli to NSCs, since electrical stimulation can affect the migration, differentiation, and proliferation of neural stem cells ${ }^{12,25}$. Electrically conductivity of scaffold can be achieved by utilization of conductive polymers and carbon based materials which include carbon nanotubes (CNTs), graphite and graphene ${ }^{14}$. Among these conductive materials, graphene, has shown novel and superior electrical, chemical and thermal properties in many research areas ${ }^{26}$. For example, unlike conductive polymers, whose conductivities easily deteriorate in harsh environments, the conductivity of graphene remains stable ${ }^{27}$ and graphene as nonresorbable materials do not present toxic byproducts ${ }^{9}$. In fact, previous report has shown $2 \mathrm{D}$ graphene film could work as a transparent electrode to electrically stimulate cells ${ }^{10}$, but its planar structure limited effective area for charge transport ${ }^{20}$. The cyclic voltammetry results indicated that it is safe for 3D-GF to electrically stimulate cells via capacitive charge injection in the potential window range from -0.2 to $+0.8 \mathrm{~V}$ (Supplementary Figure S10), just as 2D graphene film electrode. Nevertheless, owing to its larger specific surface area, porous 3D-GFs could provide a much stronger charge injection ability than $2 \mathrm{D}$ graphene films with the same geometrical area. As a result, the 3D architecture of GFs can effiectively improve the electrical stimulation performance of conductive scaffold.

In summary, we demonstrated the first utilization of 3D-GFs as a robust scaffold for NSC culture in vitro. It was found that 3D-GFs can not only support NSC growth, but also keep cells at a more active proliferation state with upregulation of Ki67 expression than that of 2D graphene films. Meanwhile, 3D-GFs can enhance the NSC differentiation towards astrocytes and especially neurons. Furthermore, 3D-GFs were proved as an efficient conductive platform to mediate electrical stimulation for differentiated NSCs. Our findings implicate the great potential of 3D-GFs in NSC research, neural tissue engineering and neural prostheses.

\section{Methods}

Fabrication of 3D-GFs and 2D graphene films for NSCs culture. The 3D-GFs were synethesized by chemical vapor deposition method with Ni foam as template (PPI 100 \pm 10 , density $320 \pm 25 \mathrm{~g} / \mathrm{m}^{2}$, Alantum Advanced Technology Materials, China) and graphene films were obtained with $\mathrm{Cu}$ foils ( $25 \mu \mathrm{m}$ thick, Alfa Aesar, USA) as the substrate (see supplementary method), according to the previous reports ${ }^{5,22}$. The samples were firstly put into a $\mathrm{FeCl}_{3}(1 \mathrm{M})$ solution for at least $72 \mathrm{~h}$ at room temperature. Then the as-prepared $3 \mathrm{D}-\mathrm{GFs}$ and $2 \mathrm{D}$ films were rinsed sequentially with $1 \mathrm{M}, 0.1 \mathrm{M}$ and $0.01 \mathrm{M} \mathrm{HCl}$ solutions, followed by rinsing with running water for at least $72 \mathrm{~h}$ to remove the etching agents. After sterilization by $75 \%$ alcohol, the 3D-GFs and 2D films were successively soaked into sterilized PBS buffer and coated with laminin $\left(5 \mu \mathrm{g} / \mathrm{mL}\right.$, Sigma-Aldrich, USA) solution in PBS for at least $4 \mathrm{~h}$ at $37^{\circ} \mathrm{C}$. Just before cell seeding, 3D-GFs \& 2D graphene films were soaked in proliferation medium overnight.

Characterization. The morphology of the samples was characterized by SEM (Quanta 400 FEG, FEI, USA). The crystallinity and number of the layer presented within graphene were examined by Raman spectrometer (lamRAM HR800, HORIBA, France). The surface chemistry was examined by XPS (Axis Ultra DLD, Kratos, UK) utilizing an $\mathrm{Al} \mathrm{K} \alpha \mathrm{X}$-ray source operated at $40 \mathrm{eV}$. For SEM imaging, cells were prepared by fixation in $2.5 \%$ glutaraldehyde in $\mathrm{pH} 7.4$ phosphate buffer, followed by post-fixation in $1 \%$ osmium tetroxide and by progressive dehydration in ethanol.

Culture of NSCs. The NSCs were isolated and purified from hippocampus of postnatal day 1 ICR mouse. For proliferation studies, NSCs were seeded at a concentration of $\sim 5 \times 10^{4} \mathrm{~mL}^{-1}$ in proliferative medium containing DMEM-F12 with 2\% B27 supplement (Life Technologies, USA), EGF ( $20 \mathrm{ng} / \mathrm{mL}$, R\&D Systems, USA) and FGF-2 (20 ng/mL, R\&D Systems, USA). Differentiation of NSCs was induced by exchanging the proliferative medium with medium containning DMEMF12, 2\% B27 supplement, retinoic acid (RA, $1 \mu \mathrm{M}$, Sigma-Aldrich, USA) and fetal bovine serum (FBS, 1\%, Life Technologies, USA) ${ }^{28}$. For cell seeding, neurospheres were collected enzymatically digested by TryplE (Life Technologies, USA) to obtain single cell suspension. After seeding, the 3D-GF scaffold together with seeded NSCs were transferred to cell culture plates on shaking table for the initial 1 day culture. All NSCs used in this study were between passage 5 and 10
SEM observation of NSCs cultured on GFs. For SEM imaging, cells were prepared by fixation in $2.5 \%$ glutaraldehyde in $\mathrm{pH} 7.4$ phosphate buffer, followed by postfixation in $1 \%$ osmium tetroxide and by progressive dehydration in ethanol.

Cell viability assay. NSCs were seeded into GF slices with $1.7 \mathrm{~mm}$ thickness, after 5 days culture, cell viability assay was performed using LIVE/DEAD ${ }^{\circledR}$ viability/ cytotoxicity kit for mammalian cells (Invitrogen, USA) according to the manufacturer instructions. Percentage of living cell was calculated by determining the percentage of calcein-AM positive cells over total cell number.

Immunofluorescence staining of NSCs. Cells were washed with PBS, fixed in 4\% paraformaldehyde for $45 \mathrm{~min}$, blocked and permeabilized for $90 \mathrm{~min}$. Primary antibodies were incubated for $90 \mathrm{~min}$, and secondary antibodies were incubated for $60 \mathrm{~min}$, followed by DAPI staining. Antibody panel used includes primary antibodies against nestin, GFAP, Ki-67 (Abcam, USA), Tuj-1 and O4 (Sigma-Aldrich, USA).

Western blot. Cells were harvested with 0.25\% trypsin in 0.03\% EDTA and lysed in RIPA buffer. Collected protein were loaded on a $10 \%$ polyacrylamide gel, separated by gel electrophoresis, and transferred onto a PVDF membrane (Millipore, USA). The membranes were blocked and incubated with primary antibodies against nestin, Tuj1, GFAP and RIP (Santa Cruz Biotechnology, USA), followed by HRP-conjugated secondary antibodies (Sigma-Aldrich, USA). The membranes were then reacted with ECL western blot substrate kit (Pierce, USA) before exposure. GAPDH was used as an internal control.

Electrical stimulation \& calcium imaging. To enable NSCs seeding on the GF substrate, a cloning ring (O.D.10 $\mathrm{mm} \&$ height $10 \mathrm{~mm}$ ) is mounted on the substrate. An electrical lead was attached directly the dry area of the GF substrate by using silver paste and copper tape. The input stimulation was applied with the aid of a function generator (S3K, Grass Technologies, USA) that gave flexibility in the stimulus signal to be applied. Before eletrical stimulatoin, the cells were loaded with Fluo-4 AM dye ( $2.5 \mu \mathrm{mol}$, Dojindo Laboratories, Japan). A series of $1 \sim 100 \mathrm{~ms}$ monophasic cathodic pulses were applied with intervals of $10 \mathrm{~s}$, the stimulation threshold was $20-30 \mu \mathrm{A}$ Time-lapse calcium level in live NSCs was imaged using fluorescence microscopy.

Statistical analysis. The Data are presented as mean \pm standard error (s. e. m.). Twogroup comparisons were tested by Student's test. * represented p value $<0.05$, $* *$ p value $<0.01$

1. Gage, F. H. Mammalian neural stem cells. Science 287, 1433-1438 (2000).

2. Rossi, F. \& Cattaneo, E. Neural stem cell therapy for neurological diseases: dreams and reality. Nat. Rev. Neurosci. 3, 401-409 (2002).

3. Lutolf, M. P., Gilbert, P. M. \& Blau, H. M. Designing materials to direct stem-cell fate. Nature 462, 433-441 (2009).

4. Lovat, V. et al. Carbon nanotube substrates boost neuronal electrical signaling. Nano Lett. 5, 1107-1110 (2005).

5. Chen, Z. et al. Three-dimensional flexible and conductive interconnected graphene networks grown by chemical vapour deposition. Nat. Mater. 10, 424-428 (2011).

6. Yavari, F. et al. High sensitivity gas detection using a macroscopic threedimensional graphene foam network. Sci. Rep. 1, 166 (2011).

7. Ji, H. et al. Ultra-thin graphite foam: a three-dimensional conductive network for battery electrodes. Nano Lett. 12, 2446-2451 (2012).

8. Yong, Y.-C., Dong, X.-C., Chan-Park, M. B., Song, H. \& Chen, P. Macroporous and monolithic anode based on polyaniline hybridized three-dimensional graphene for high-performance microbial fuel cells. ACS Nano 6, 2394-2400 (2012).

9. Nayak, T. R. et al. Graphene for controlled and accelerated osteogenic differentiation of human mesenchymal stem cells. ACS Nano 5, 4670-4678 (2011).

10. Park, S. Y. et al. Enhanced differentiation of human neural stem cells into neurons on graphene. Adv. Mater. 23, H263-H267 (2011).

11. Wang, Y. et al. Fluorinated graphene for promoting neuro-induction of stem cells. Adv. Mater. 24, 4285-4290 (2012)

12. Chang, K.-A. et al. Biphasic electrical currents stimulation promotes both proliferation and differentiation of fetal neural stem cells. PLOS ONE 6, e18738 (2011).

13. Ghasemi-Mobarakeh, L. et al. Application of conductive polymers, scaffolds and electrical stimulation for nerve tissue engineering. J. Tissue. Eng. Regen. Med. 5, e17-e35 (2011)

14. Kotov, N. A. et al. Nanomaterials for neural interfaces. Adv. Mater. 21, 3970-4004 (2009).

15. Ferrari, A. C. et al. Raman spectrum of graphene and graphene layers. Phys. Rev. Lett. 97, 187401 (2006).

16. Huang, X. et al. Functional nanoporous graphene foams with controlled pore sizes. Adv. Mater. 24, 4419-4423 (2012).

17. Georgakilas, V. et al. Functionalization of graphene: covalent and non-covalent approaches, derivatives and applications. Chem. Rev. 112, 6156-6214 (2012).

18. Li, N. et al. The promotion of neurite sprouting and outgrowth of mouse hippocampal cells in culture by graphene substrates. Biomaterials 32, 9374-9382 (2011). 
19. Scholzen, T. \& Gerdes, J. The Ki-67 protein: from the known and the unknown. J. Cell. Physiol. 182, 311-322 (2000).

20. Wang, K., Fishman, H. A., Dai, H. \& Harris, J. S. Neural stimulation with a carbon nanotube microelectrode array. Nano Lett. 6, 2043-2048 (2006).

21. Li, X. et al. Fabrication of density gradients of biodegradable polymer microparticles and their use in guiding neurite outgrowth. Adv. Funct. Mater. 20, $1632-1637$ (2010)

22. Li, X. et al. Large-area synthesis of high-quality and uniform graphene films on copper foils. Science 324, 1312-1314 (2009).

23. Bendali, A. et al. Purified neurons can survive on peptide-free graphene layers. Adv. Healthcare Mater. DOI:10.1002/adhm.201200347 (2013).

24. Béduer, A. et al. Engineering of adult human neural stem cells differentiation through surface micropatterning. Biomaterials 33, 504-514 (2012).

25. Huang, Y.-J., Wu, H.-C., Tai, N.-H. \& Wang, T.-W. Carbon nanotube rope with electrical stimulation promotes the differentiation and maturity of neural stem cells. Small 8, 2869-2877 (2012).

26. Geim, A. K. \& Novoselov, K. S. The rise of graphene. Nat. Mater. 6, 183-191 (2007).

27. Dong, X. et al. 3D graphene foam as a monolithic and macroporous carbon electrode for electrochemical sensing. ACS Appl. Mater. Interfaces 4, 3129-3133 (2012).

28. Takahashi, J., Palmer, T. D. \& Gage, F. H. Retinoic acid and neurotrophins collaborate to regulate neurogenesis in adult-derived neural stem cell cultures. J. Neurobiol. 38, 65-81 (1999).

\section{Acknowledgements}

This work was funded by MOST (Grant No. 2011CB965004), NSFC (Grant No.31101059), "Strategic Priority Research Program" from CAS (Grant No. XDA01030200) and Open Project Program of State Key Laboratory of Molecular Engineering of Polymers (Fudan University, Grant No. K2012-09). We thank Dr. Zheng Yin, Chen Xie, Mengdi Li, Ziyun Jiang, Dr. Tao Kong, Dr. Lin Qi, and Dr. Luhua Lu for their assistances.

\section{Author contributions}

N.L., Q.Z., J.D., M.T. and G.C. conceived and designed the experiments. Q.Z., S.G. and L.L. prepared the samples and performed characterization. N.L. and L.W. involved the biological experiments. Q.S. performed electrochemical analysis. R.H. performed SEM experiments and analysis. N.L., Q.Z., J.D., M.T. and G.C. were mainly responsible for preparing the manuscript with further inputs from other authors. All the authors discussed the results and commented on the manuscript.

\section{Additional information}

Supplementary information accompanies this paper at http://www.nature.com/ scientificreports

Competing financial interests: The authors declare no competing financial interests.

License: This work is licensed under a Creative Commons

Attribution-NonCommercial-NoDerivs 3.0 Unported License. To view a copy of this license, visit http://creativecommons.org/licenses/by-nc-nd/3.0/

How to cite this article: Li, N. et al. Three-dimensional graphene foam as a biocompatible and conductive scaffold for neural stem cells. Sci. Rep. 3, 1604; DOI:10.1038/srep01604 (2013). 